\title{
Двумерные брэгговские резонаторы на основе планарных диэлектрических волноводов (от теории к модельному тестированию)
}

\author{
(ㄱ Н.С. Гинзбург, Н.Ю. Песков, В.Ю. Заславский, Е.Р. Кочаровская , А.М. Малкин, \\ А.С. Сергеев, В.Р. Барышев, М.Д. Проявин, Д.И. Соболев
}

Институт прикладной физики Российской академии наук, 603950 Нижний Новгород, Россия

ฯ E-mail: katya@appl.sci-nnov.ru

Поступила в Редакцию 24 апреля 2019 г.

В окончательной редакции 29 апреля 2019 г.

Принята к публикации 29 апреля 2019 г.

В рамках аналитического рассмотрения и трехмерного моделирования с использованием кода CST Microwave Studio выполнен теоретический анализ электродинамических характеристик двумерных брэгговских резонаторов на основе планарных диэлектрических волноводов с двоякопериодической гофрировкой. Подобные резонаторы представляют значительный интерес для получения направленного узкополосного излучения в гетеролазерах с большими размерами активной зоны. Проведены модельные электродинамические эксперименты по „холодному“ тестированию указанных структур в миллиметровом диапазоне. Показано хорошее соответствие экспериментальных результатов с результатами моделирования, включая существование наиболее высокодобротной моды внутри полосы брэгговских отражений в отсутствие дефектов периодичности.

Ключевые слова: планарные диэлектрические волноводы, двумерная распределенная обратная связь, гетеролазеры.

DOI: 10.21883/FTP.2019.10.48283.29

\section{1. Введение}

В технике полупроводниковых лазеров (в том числе гетеролазеров) широкое распространение получили брэгговские резонаторы, реализуемые на основе диэлектрических структур с периодической вариацией либо показателя преломления, либо толщины волноведущего слоя. В таких структурах имеет место взаимное брэгговское рассеяние двух встречно-распространяющихся волн, обеспечивающее реализацию распределенной обратной связи (РОС). Достоинствами брэгговских резонаторов являются возможность селекции мод по продольному индексу, облегчающая задачу генерации монохроматического излучения, а также компактность, обусловленная отсутствием внешних отражающих элементов [1-6].

Однако в случае активных сред с большими латеральными размерами связь продольно-распространяющихся встречных волн не обеспечивает селекцию по поперечным индексам. В результате ширины РОС лазеров в условиях поддержания одномодовой узкополосной генерации ограничены несколькими длинами волн. При дальнейшем увеличении поперечных размеров спектр генерируемого излучения усложняется. Одновременно, вследствие нарушения пространственной когерентности излучения, расширяется диаграмма направленности $[7,8]$. Указанные ограничения на размеры структур естественным образом ограничивают объем активной среды и, соответственно, полную мощность излучения.
В настоящее время реализованы как гетеролазеры на межзонных переходах, так и квантово-каскадные лазеры, латеральный размер которых достигает сотен длин волн [7-10]. В этой связи актуальной является разработка схем полупроводниковых лазеров, способных обеспечить когерентность и синхронизацию излучения активных сред с большими латеральными размерами. Важно подчеркнуть, что для таких лазеров, в частности гетеролазеров, вследствие эпитаксиальнолитографической технологии изготовления типична планарная геометрия.

Одним из возможных путей решения указанной задачи является применение так называемой двумерной (2D) РОС, которая может быть реализована в брэгговских структурах планарной геометрии с двоякопериодической модуляцией толщины волноводного диэлектрического слоя. Как показано в [11-13], использование такого вида РОС позволяет синхронизовать излучение активной среды, характеризующейся большими значениями параметра Френеля по двум поперечным направлениям (рис. 1):

$$
\mathrm{F}_{x}=l_{x}^{2} / \lambda l_{z} \gg 1, \quad \mathrm{~F}_{z}=l_{z}^{2} / \lambda l_{x} \gg 1 .
$$

Следует отметить, что к настоящему времени эффективность использования двумерной РОС подтверждена экспериментально при реализации лазеров на свободных электронах [14], запитываемых мощными релятивистскими электронными пучками ленточной геометрии. 


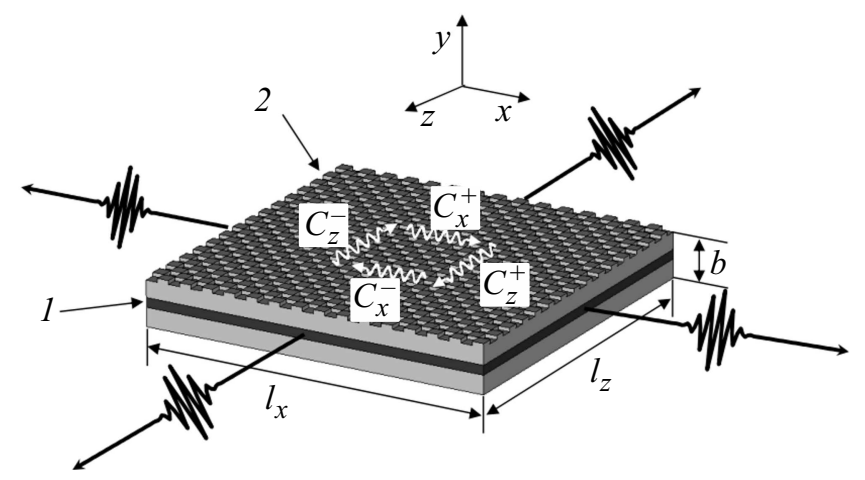

Рис. 1. Общая схема гетеролазера с двумерной распределенной обратной связью: 1 - активная среда, 2 - двумернопериодический брэгговский резонатор на основе планарного диэлектрического волновода с шахматной гофрировкой. Волнистыми стрелками показаны направления парциальных волновых потоков, связанных на 2D брэгговской структуре.

В этом случае брэгговские структуры представляли собой полые планарные волноводы с двоякопериодической гофрировкой боковых металлических стенок. В результате в 4-миллиметровом диапазоне длин волн получена узкополосная генерация с рекордно высоким для указанного диапазона уровнем мощности до $100 \mathrm{MBT}$.

В настоящей работе проведен теоретический анализ 2D брэгговских структур на основе диэлектрических волноводов в проекции на экспериментальное тестирование их электродинамических характеристик. Ввиду значительных технологических проблем с изготовлением 2D брэгговских структур для ИК-диапазонов (с характерными периодами в доли микрон) экспериментальное доказательство работоспособности подобных структур проведено в коротковолновой части миллиметрового диапазона, где указанные структуры были изготовлены на основе 3D печати. Тестирование проведено на специализированном электродинамическом стенде, обеспечивающем формирование широких волновых пучков с плоским фазовым фронтом. Показано хорошее соответствие измеренных электродинамических характеристик с расчетными. Следует отметить, что продуктивность метода модельного тестирования в микроволновом диапазоне различных типов периодических структур (или фотонных кристаллов), предназначенных для работы в ИК и оптических диапазонах, продемонстрировано ранее, в частности в работах [15-18].

\section{2. Моделирование селективных характеристик двумерных брэгговских резонаторов}

Рассмотрим следующую модель 2D брэгговского резонатора, предполагая, что на прямоугольный участок одной из поверхностей диэлектрической пластины нанесена двумерно-периодическая гофрировка, при которой толщина пластины промодулирована по закону (рис. 1)

$$
b(x, z)=b_{0}+b_{1}\left(\cos \left(\bar{h}_{x} x+\bar{h}_{z} z\right)+\cos \left(\bar{h}_{x} x-\bar{h}_{z} z\right)\right),
$$

где $\bar{h}_{x}=\bar{h}_{z}=2 \pi / d_{x, z}$ - проекции трансляционных векторов $\stackrel{1}{K} \pm=\bar{h}_{x} \underset{x_{0}}{\stackrel{\mathrm{r}}{d}} \bar{h}_{z} \underset{z_{0}}{\mathrm{r}}$ двумерной решетки на $x$ и $z$ направления, $d_{x, z}$ - периоды соответствующих модуляций. Участок с модуляцией имеет длину $l_{z}$ по продольной оси $z$ и ширину $l_{x}$ по поперечной (латеральной) координате $x$. Указанный участок формирует 2D брэгговский резонатор. Заметим, что для практической реализации синусоидальная гофра (2) может быть заменена на шахматную $[12,19]$.

Поля собственных ТМ-поляризованных мод 2D брэгговского резонатора [13] задаются как сумма 4 парциальных волновых потоков

$$
\begin{aligned}
E_{y}= & \operatorname{Re}\left(f ( y ) \left(C_{z}^{+}(x, z) e^{-i \bar{h} z}+C_{z}^{-}(x, z) e^{i \bar{h} z}\right.\right. \\
& \left.\left.\left.+C_{x}^{+}(x, z) e^{-i \bar{h} x}+C_{x}^{-}\right)(x, z) e^{i \bar{h} x}\right) e^{i \omega t}\right),
\end{aligned}
$$

распространяющихся в направлениях $\pm x$ и $\pm z$. Здесь $C_{x, z}^{ \pm}$- медленно меняющиеся в масштабе длины волны комплексные амплитуды, функция $f(y)$, определяющая структуры волн в направлении, нормальном к поверхности волновода, совпадает с одной из ТМ-мод указанного волновода, $E_{y}-y$-компонента электрического поля. При этом средняя толщина волновода подбирается таким образом, чтобы в рабочем диапазоне частот распространялась единственная мода.

На брэгговской структуре, заданной соотношением (2), каждый из волновых потоков в условиях брэгговского резонанса рассеивается в двух взаимно противоположных направлениях, каждое из которых перпендикулярно исходному направлению распространения. Взаимное рассеяние волновых потоков в указанных условиях описывается уравнениями [11-13]

$$
\begin{aligned}
& \pm \frac{\partial C_{z}^{ \pm}}{\partial z}+i \delta C_{z}^{ \pm}+i \alpha \bar{h}\left(C_{x}^{+}+C_{x}^{-}\right)=0 \\
& \pm \frac{\partial C_{x}^{ \pm}}{\partial x}+i \delta C_{x}^{ \pm}+i \alpha \bar{h}\left(C_{z}^{+}+C_{z}^{-}\right)=0
\end{aligned}
$$

где $\delta=h(\omega)-\bar{h}-$ расстройка брэгговского резонанса,

$$
\begin{aligned}
\alpha & =\frac{c \bar{h} b_{1}}{4 \omega} \\
& \times \frac{\left(\varepsilon-\bar{h}^{2} c^{2} / \omega^{2}\right)\left(1+\varepsilon^{-2}\right)}{\left(\bar{h}^{2} c^{2} /\left(\varepsilon \omega^{2}\right)+\bar{h}^{2} c^{2} / \omega^{2}+1\right) b_{0}+2\left(\bar{h}^{2}-\omega^{2} / c^{2}\right)^{-1 / 2}}
\end{aligned}
$$

- коэффициент связи парциальных волн $[12,13]$. Заметим, что волновое число и частота $\omega$ связаны законом дисперсии низшей моды диэлектрического волновода. Следует также отметить, что при выводе (3) пренебрегается дифракцией волновых потоков, в предположении, что соответствующие параметры Френеля достаточно велики. 
Спектр собственных мод 2D брэгговского резонатора может быть найден из решения краевой задачи, для чего уравнения (3) следует дополнить граничными условиями

$\left.C_{z}^{+}\right|_{z=0}=0,\left.\quad C_{z}^{-}\right|_{z=l_{z}}=0,\left.\quad C_{x}^{+}\right|_{x=0}=0,\left.\quad C_{x}^{-}\right|_{x=l_{x}}=0$,

которые соответствуют отсутствию внешних потоков энергии.

В условиях сильной связи волн $\alpha l_{x, z} \gg 1$ решения для комплексных $\delta_{n, m}$, которые определяют спектр собственных частот

$$
\omega_{n, m} \approx c \bar{h}+c \operatorname{Re} \delta_{n, m}
$$

и добротностей мод 2D брэгговского резонатора

$$
Q_{n, m} \approx \bar{h} /\left(2 \operatorname{Im} \delta_{n, m}\right),
$$

даются соотношениями [11-13]

$$
\begin{gathered}
\delta_{n, m}= \pm \frac{\pi^{2} m n}{2 \alpha l_{z} l_{x}}+i \frac{\pi^{2}}{2 \alpha^{2} l_{z} l_{x}}\left(\frac{n^{2}}{l_{z}}+\frac{m^{2}}{l_{x}}\right), \\
\delta_{n, m}= \pm\left[2 \alpha+\frac{\pi^{2}}{4 \alpha}\left(\frac{n^{2}}{l_{z}^{2}}+\frac{m^{2}}{l_{x}^{2}}\right)\right]+i \frac{\pi^{2}}{2 \alpha^{2}}\left(\frac{n^{2}}{l_{z}^{3}}+\frac{m^{2}}{l_{x}^{3}}\right),
\end{gathered}
$$

где $n$ и $m-$ индексы мод по продольной $z$ и поперечной $x$ координатам соответственно. Согласно (7), существует два семейства высокодобротных мод (рис. 2,a). Одно из них (семейство „I“ на рис. 2, $a$, формула (7 a)) расположено вблизи брэгговской частоты, второе (семейство „ІІ“价, формула (7b)) - вблизи границы зоны запирания. Двумерная брэгговская структура обладает селективностью по обоим индексам $n$ и $m$. При $l_{z}>l_{x}$ максимальной добротностью обладает мода из семейства „I“ с номерами $n=1, m=0$. Важно подчеркнуть, что частота указанной моды лежит внутри полосы брэгговских отражений в отсутствие дефектов периодичности [20]. Это обстоятельство кардинально отличает 2D брэгговские резонаторы от одномерных прототипов [1-3].

Для верификации применимости аналитической модели проведено полное трехмерное моделирование указанных систем на основе коммерческого кода CST Microwave Studio. Моделирование проводилось для структуры с диэлектрической проницаемостью $\varepsilon=2.4$ и тангенсом угла потерь $\tan \delta=0.002$, экспериментально измеренным в „холодных“ тестах для пластиков типа SBS (Sterol-Butadiene-Sterol). Структура с периодами $d_{x}=d_{z}=4$ мм, толщиной $b_{0}=1$ мм, продольным и поперечным размерами $l_{z}=l_{x}=180$ мм, обеспечивающая связь низших ТМ-волн, была размещена на металлической подложке. Двоякопериодическая модуляция была нанесена на внешнюю поверхность диэлектрика. Найденная в „СST“моделировании частота наиболее добротной 2моды ( $\sim 60$ ГГц) и абсолютное значение добротности находится в хорошем соответствии с результатами аналитической теории (рис. $2, b$ ). При этом моделирование
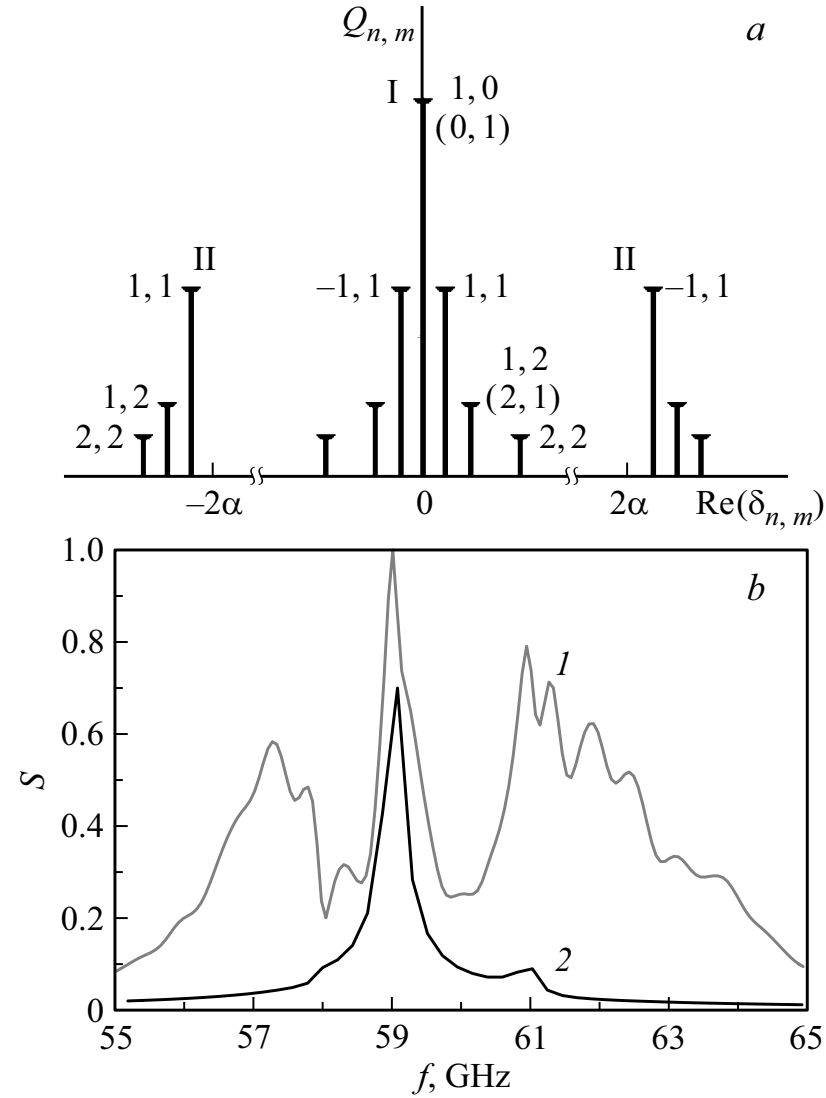

Pис. 2. $a-$ спектр мод двумерного брэгговского резонатора, найденный в рамках метода связанных волн (формула (7)). $b-$ результаты CST моделирования возбуждения двумерной брэгговской структуры коротким электромагнитным импульсом $\omega_{0} \Delta t \approx 40$. Спектры поля на полном интервале моделирования $\omega_{0} t \approx 0-6000$ (серая кривая 1 ) и на конечной стадии процесса затухания возмущений $\omega_{0} t \approx 4000-6000$ (черная кривая 2), на которой происходит выделение моды с минимальными потерями на излучение; $l_{x}=l_{z}=45 \lambda, b_{0}=0.25 \lambda$, $\varepsilon=2.4$.

подтверждает наличие в двумерном брэгговском резонаторе высокодобротных мод в центре полосы непрозрачности. Таким образом, как аналитическая теория, так и прямое численное моделирование показывают, что двумерная двоякопериодическая структура, нанесенная на поверхность диэлектрика, обеспечивает эффективную селекцию мод по двум координатам. Следует отметить, что трехмерная модель соответствует экспериментальному макету, описанному в разд. 3 (см. рис. 3 ).

На рис. 4 (пунктирные кривые) представлены частотные зависимости коэффициента прохождения

$$
T=\frac{\int_{0}^{l_{x}}\left|C_{z}^{+}\left(x, l_{z}\right)\right|^{2} d x}{\int_{0}^{l_{x}}\left|C_{z}^{+}(x, 0)\right|^{2} d x}
$$

падающего излучения для случая нормального и наклонного падения, полученные в рамках 3D моделирования. 


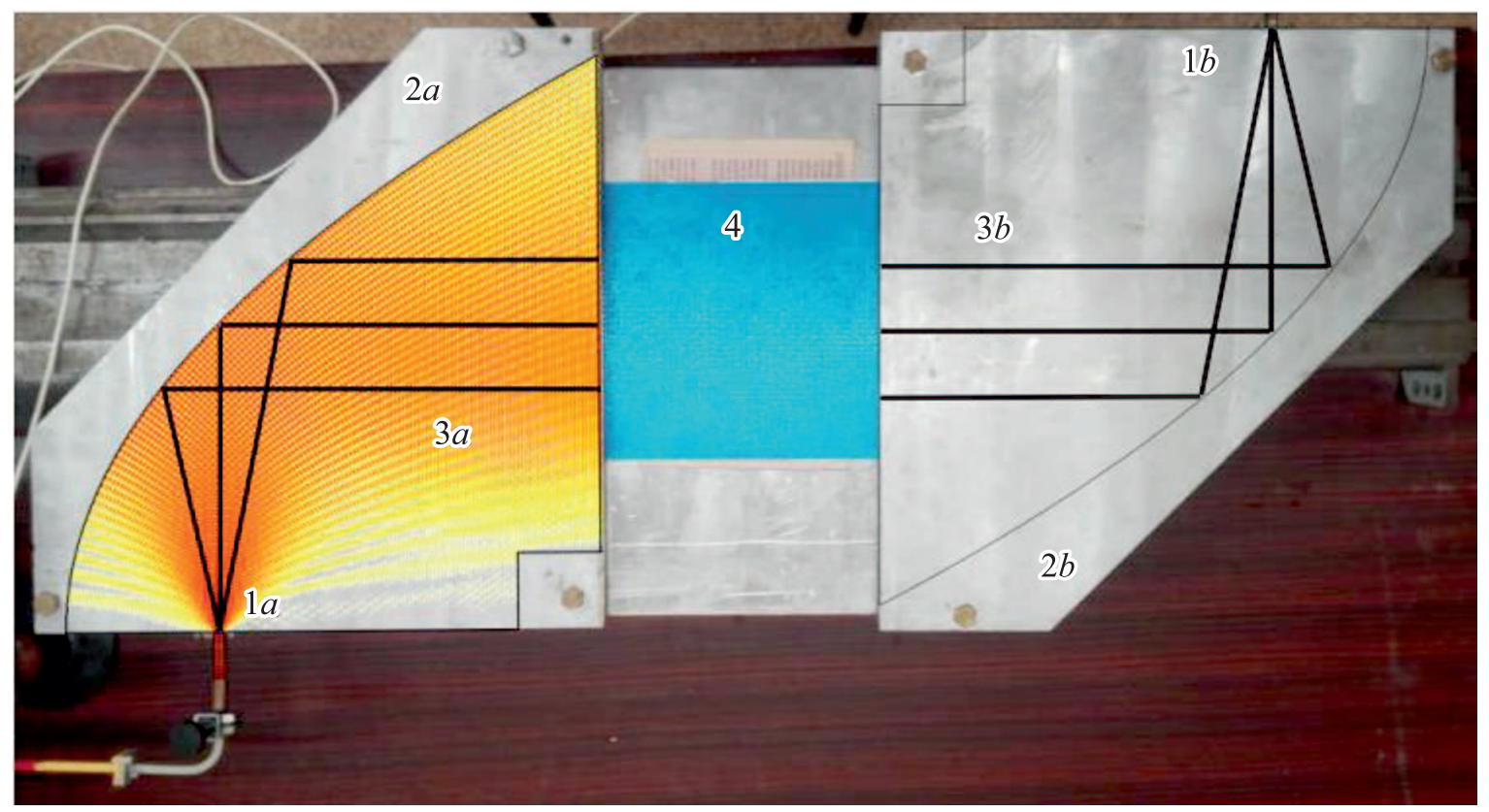

Рис. 3. Стенд для измерения электродинамических характеристик двумерных брэгговских структур: 1 - одномодовые волноводные тракты, один из которых запитывается панорамным источником; 2 - квазиоптические линии; формирующие волновой пучок с плоским фазовым фронтом; 3 - падающий $(a)$ и прошедший $(b)$ волновые пучки; 4 - двумерно-периодическая брэгговская структура на основе планарного диэлектрического волновода. Полупрозрачным слоем показано распределение электрического поля внутри формирующей линии.

Относительная ширина полосы брэгговского рассеяния хорошо согласуется с оценкой $4 \alpha$, вытекающей из формулы $(7 b)$. В случае наклонного падения возникает просветление на частоте, близкой к брэгговской, которое, как показано в $[12,19,20]$, обусловлено возбуждением основной фундаментальной моды системы, обладающей максимальной добротностью.

\section{3. Экспериментальное исследование диэлектрических двумерных брэгговских структур в миллиметровом диапазоне}

Исследование электродинамических характеристик указанных структур проводилось в „холодных“ электродинамических тестах в диапазоне 60 ГГц. Планарные диэлектрические волноводы с нанесенной на поверхность двоякопериодической шахматной гофрировкой („,4“ на рис. 3) изготавливались с помощью 3D принтеров из пластиков типа SBS. В серии предварительных экспериментов у данных пластиков были определены диэлектрическая проницаемость $\varepsilon \approx 2.3-2.5$ и тангенс потерь $\tan \delta \approx 0.002$ [21]. На основе разработанной технологии были изготовлены макеты двумерных диэлектрических структур с геометрическими размерами $180 \times 180 \mathrm{MM}$ (т.е. около $45 \times 45$ длин волн в рабочем диапазоне частот), толщина структур составляла 1 мм, период и глубина гофрировки 4 и 0.4 мм соответственно.
Возбуждение планарной диэлектрической 2D брэгговской структуры осуществлялось широким волновым пучком с плоским фазовым фронтом, который формировался с помощью планарной квазиоптической передающей линии. Подобная линия представляла собой параллельные металлические пластины с заключенным между ними параболическим зеркалом, в фокусе которого располагался одномодовый волновод. Падающее излучение генерировалось с помощью панорамного высокочастотного источника P2-67 в виде $H_{0,1}$ волны прямоугольного волновода. На выходе линии с шириной $375 \mathrm{Mм}$ и зазором 5 мм формировался плоский волновой пучок со структурой близкой к ТЕМ-волне планарного волновода (см. рис. 3,a). Меньшая по размеру брэгговская структура $(180 \times 180$ мм $)$ располагалась в центральной части передающей линии, где поперечное распределение падающего волнового пучка близко к однородному. Аналогичная линия передачи использовалась в качестве приемника прошедшего (рассеянного) через структуру излучения при измерении коэффициента прохождения (см. рис. 3).

Согласно проведенным расчетам, исследуемая структура имела рабочую зону брэгговского рассеяния в районе 60 ГГц. Результаты измерений частотных зависимостей коэффициентов прохождения изготовленного макета диэлектрической 2D структуры представлены на рис. 4 (сплошные кривые). Как видно из сравнения с результатами моделирования, эксперименты продемонстрировали хорошее соответствие с расчетами как по 

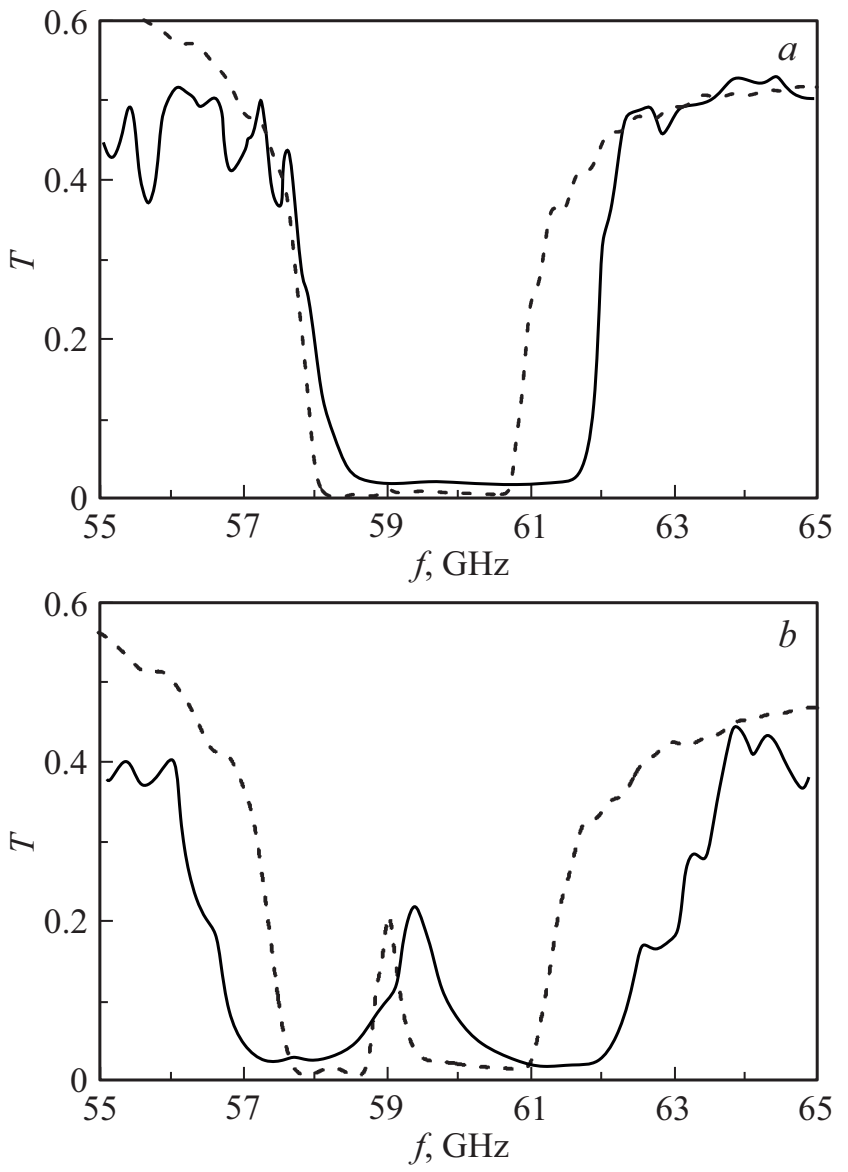

Рис. 4. Результаты CST моделирования (пунктир) и экспериментального исследования (сплошные линии) частотных зависимостей коэффициентов прохождения $T$ диэлектрической структуры с двумерной шахматной гофрировкой в случае $(a)$ „нормального“ падения и $(b)$ „наклонного“ падения волнового пучка с плоским фазовым фронтом. В последнем случае видно просветление системы в центре полосы непрозрачности, обусловленное возбуждением основной моды резонатора.

положению и ширине брэгговской полосы, так и по амплитудным характеристикам интегральных коэффициентов рассеяния.

Следует также заметить, что в проводимых экспериментах на входе планарной квазиоптической передающей линии использовался одномодовый прямоугольный волновод, имеющий достаточно широкую диаграмму направленности излучения. Вследствие чего, согласно результатам моделирования, на выходе формирующей линии возникала интерференция лучей, как отраженных от параболического зеркала, так и излучаемых непосредственно из волновода. Данный эффект приводил к пространственной модуляции волнового пучка, падающего на 2D структуру (рис. 3). Этим может быть объяснена наблюдаемая в эксперименте некоторая изрезанность интегральных характеристик рассеянного на двумерной решетке излучения.

\section{4. Заключение}

Таким образом, проведенные теоретические и экспериментальные исследования подтвердили реализуемость и работоспособность двумерно-периодических брэгговских структур на основе планарных диэлектрических волноводов. В модельных экспериментах, проведенных в миллиметровом диапазоне, показана адекватность теоретических моделей для описания таких структур. Продемонстрировано, что двумерно-периодические брэгговские резонаторы позволяют обеспечить селекцию мод по двум индексам при длине и ширине системы, на порядки превышающих длину волны. Тем самым показана перспективность использования таких структур для получения узкополосного узконаправленного излучения в полупроводниковых лазерах с большими размерами активной зоны, включая гетеролазеры и квантовокаскадные лазеры.

\section{Финансирование работы}

Работа выполнена в рамках государственного задания ИПФ РАН на проведение научных исследований по теме № 0035-2019-0001 и проекта Российского фонда фундаментальных исследований № 18-48-520022.

\section{Конфликт интересов}

Авторы заявляют, что у них нет конфликта интересов.

\section{Список литературы}

[1] A. Yariv. Quantum Electronics (N.Y., Wiley, 1975).

[2] H. Kogelnik, C.V. Shank. J. Appl. Phys., 43, 2327 (1972).

[3] H. Kogelnik. Theory of dielectric waveguides. In: Integrated Optics (Springer Berlin, Heidelberg, 1979) v. 7.

[4] H. Ghafouri-Shiraz. Distributed Feedback Laser Diodes and Optical Tunable Filters (N.Y., Wiley, 2003) гл. 2.

[5] G. Morthier, R.J. Baets. Lightwave Technol., 9, 1305 (1991).

[6] А.А. Афанасьев, С.Ю. Михневич. Квант. электрон., 34, 315 (2004).

[7] D.A. Yanson, E.U. Rafailov, G.S. Sokolovskii, V.I. Kuchinskii, A.C. Bryce, J. Marsh, W. Sibbett. J. Appl. Phys., 95, 1502 (2004).

[8] Г.С. Соколовский, В.В. Дюделев, И.М. Гаджиев, С.Н. Лосев, А.Г. Дерягин, В.И. Кучинский, Э.У. Рафаилов, В. Сиббет. Письма ЖТФ, 31, 28 (2005).

[9] В.Я. Алешкин, Б.Н. Звонков, С.М. Некоркин, Вл.В. Кочаровский. ФТП, 39, 171 (2005).

[10] S.M. Nekorkin, A.A. Biryukov, P.B. Demina, N.N. Semenov, B.N. Zvonkov, V.Ya. Aleshkin, A.A. Dubinov, V.I. Gavrilenko, K.V. Maremyanin, S.V. Morozov, A.A. Belyanin, V.V. Kocharovsky, Vl.V. Kocharovsky. Appl. Phys. Lett., 90, 171106 (2007).

[11] N.S. Ginzburg, N.Yu. Peskov, A.S. Sergeev. Optics Commun., 112, 151 (1994).

[12] В.Р. Барышев, Н.С. Гинзбург, В.Ю. Заславский, А.М. Малкин, А.С. Сергеев, М. Тумм. Квант. электрон., 39, 463 (2009). 
[13] N.S. Ginzburg, V.R. Baryshev, A.S. Sergeev, A.M. Malkin. Phys. Rev. A, 91, 053806 (2015).

[14] A.V. Arzhannikov, N.S. Ginzburg, P.V. Kalinin, S.A. Kuznetsov, A.M. Malkin, N.Yu. Peskov, A.S. Sergeev, S.L. Sinitsky, V.D. Stepanov, M. Thumm, V.Yu. Zaslavsky. Phys. Rev. Lett., 117, 114801 (2016).

[15] A. Gruss, K.T. Tam, T. Tamir. Appl. Phys. Lett., 36, 523 (1980).

[16] W.M. Robertson, G. Arjavalingam, R.D. Meade, K.D. Brom mer, A.M. Rappe, J.D. Joannopoulos. Phys. Rev. Lett., 68, 2023 (1992).

[17] J.R. James, I. Abd-Eldayem. IEE Proc. Optoelectron., 144 (4), 221 (1997).

[18] W. Freude1, G.-A. Chakam, J.-M. Brosi, C. Koos. Photonic Crystals Advances in Design (Fabrication, and Characterization, Wiley-VCH, 2004) гл. 10.

[19] Н.С. Гинзбург, Г.Г. Денисов, В.Ю. Заславский, С.В. Кузиков, Н.Ю. Песков, А.С. Сергеев, А.В. Аржанников, П.В. Калинин, С.Л. Синицкий, М. Тумм. Изв. вузов. Радиофизика, 58, 842 (2005).

[20] N.S. Ginzburg, N.Yu. Peskov, A.S. Sergeev, G.G. Denisov, S.V. Kuzikov, V.Yu. Zaslavsky, A.V. Arzhannikov, P.V. Kalinin, S.L. Sinitsky, M. Thumm. Appl. Phys. Lett., 92, 103512 (2008).

[21] N.Yu. Peskov, V.R. Baryshev, N.S. Ginzburg, E.R. Kocharovskaya, A.M. Malkin, D.M. Padozhnikov, M.D. Proyavin, V.Yu. Zaslavsky. Proc. 3rd Int. Conf. Terahertz and Microwave Radiation: Generation, Detection and Applications (TERA-2018), (Nizhny Novgorod, Russia, 2018) EPJ Web of Conferences, v. 195, art. 02012.

Редактор Г.А. Оганесян

\section{D Bragg resonators based on planar dielectric waveguides (theory and modelling experiments)}

N.S. Ginzburg, N.Yu. Peskov, V.Yu. Zaslavsky,

E.R. Kocharovskaya, A.M. Malkin, A.S. Sergeev,

V.R. Baryshev, M.D. Proyavin, D.I. Sobolev

Institute of Applied Physics,

Russian Academy of Sciences,

603950 Nizhny Novgorod, Russia

Abstract Using analytical approaches and CST Microwave Studio 3D simulations, we have undertaken a theoretical analysis of electrodynamical characteristics of 2D Bragg structures based on planar oversized dielectric waveguides with double-periodical corrugation. Such structures are of significant interest for obtaining directed narrow-band radiation in heterolasers with large dimensions of active area. Modelling electrodynamical experiments on "cold“ testing of such structures in millimeter wavelength band were conducted. Fine agreement between the experimental results and the simulation results was demonstrated which includes the presence of the highest-quality mode inside the Bragg reflection band in absence of the periodicity defects. 